This is the post-print version of the article, which has been published in Computers in Biology and Medicine 2018, 96, 274-282.

The final publication is available at

https://doi.org/10.1016/j.compbiomed.2018.04.003

\title{
The effect of percutaneous transluminal angioplasty of superficial femoral artery on pulse wave features
}

\author{
Mikko Peltokangas ${ }^{\mathrm{a}, *}$, Velipekka Suominen ${ }^{\mathrm{b}}$, Damir Vakhitov ${ }^{\mathrm{b}}$, Jarmo Verho $^{\mathrm{a}}$, \\ Janne Korhonen $^{\mathrm{c}}$, Jukka Lekkala ${ }^{\mathrm{a}}$, Antti Vehkaoja ${ }^{\mathrm{a}}$, Niku Oksala ${ }^{\mathrm{b}, \mathrm{d}}$ \\ ${ }^{a}$ BioMediTech Institute and Faculty of Biomedical Sciences and Engineering, Tampere \\ University of Technology, Tampere, Finland. Email addresses: firstname.lastname@tut.fi. \\ Postal address: Faculty of Biomedical Sciences and Engineering, Tampere University of \\ Technology, Korkeakoulunkatu 3, FI-33720 Tampere, Finland. tel. +3583311511. \\ ${ }^{b}$ Division of Vascular Surgery, Department of Surgery, Tampere University Hospital, \\ Tampere, Finland. \\ ${ }^{c}$ Division of Interventional Radiology, Department of Radiology, Tampere University \\ Hospital, Tampere, Finland \\ ${ }^{d}$ Finnish Cardiovascular Research Center Tampere, Surgery, Faculty of Medicine and Life \\ Sciences, University of Tampere, Tampere, Finland.
}

\begin{abstract}
We aimed to analyze the effects of percutaneous transluminal angioplasty (PTA) of the superficial femoral artery (SFA) on arterial pulse waves (PWs). Altogether 24 subjects i.e. 48 lower limbs were examined including 26 treated lower limbs having abnormal ankle-to-brachial pressure index $(\mathrm{ABI})(\mathrm{ABI}<0.9$ or $\mathrm{ABI}>1.3$ ) and 22 non-treated lower limbs. The measurements were conducted in pre-, peri- and post-treatment phases as well as in follow-up visit after 1 month. Both ABI and toe pressures measured by standard equipment were used as reference values. PW-derived parameters include ratios of different peaks of the PW and time differences between them as well as aging index. Both treated and non-treated limbs were compared in pre- and post-treatment as well as follow-up visit conditions. The results were evaluated in terms of statistical tests, Bland-Altman-plots, free-marginal multirater $\kappa$-analysis and multiple linear regression analysis. PTA was found to cause small changes to the studied PW-derived parameters of the treated limb which were observed immediately after the treatment, but the changes were more pronounced in the follow-up
\end{abstract}

\footnotetext{
* Corresponding author

Email address: mikko.peltokangas@tut.fi (Mikko Peltokangas)
} 
visit. In addition, we observed that the endovascular instrumentation itself does not cause significant changes to the PW-derived parameters. The results show that PW-analysis could be a useful tool for monitoring the treatment-effect of the PTA. However, because the pre-treatment differences of the treated and non-treated limb were small, further studies with subjects having no arterial diseases are required. The study demonstrates the potential of the $\mathrm{PW}$ analysis in monitoring vascular abnormalities.

Keywords: Atherosclerosis, Electromechanical sensors, Photoplethysmography, Peripheral arterial disease, Pulse wave measurements

\section{Introduction}

Atherosclerosis may be present as stiffening, stenosis or occlusion of the arteries. Peripheral arterial disease (PAD) is a specific form of atherosclerosis affecting mainly the lower limbs. PAD may be asymptomatic, but often it causes symptoms such as intermittent claudication, or conditions threatening the vitality of the limb. These conditions may be critical limb ischemia with rest pain and tissue defects and acute limb ischemia. PAD is also seen as a risk factor of acute cardiovascular events such as a stroke or myocardial infarction. PAD is treated commonly by means of percutaneous transluminal angioplasty (PTA) in which the stenosed artery is dilated in a minimally invasive endovascular treatment procedure. According to the past experience, the PTA results in the disruption of the atherosclerotic plaque and initiates the histological remodeling of the arterial wall [1]. In some cases, inward remodeling and a restenosis occur, resulting in the worsening of the symptoms. The patency rates of the PTA are reported to vary between $75 \%-97 \%$ and $60 \%-84 \%$ for one- and two-year follow-up times, respectively 2]. Commonly, the patients are subjected to a 1-month follow-up to ensure the technical success of the treatment. The re-examination includes the measurement of the ankle-to-brachial pressure in$\operatorname{dex}(\mathrm{ABI})$. However, ABI has several limitations, such as varying sensitivity and specificity from study-to-study [3] and challenges especially with the dia- 
betic and mediasclerotic patients [4, 5, 6]. As the ABI measurement has several drawbacks, imaging methods such as magnetic resonance angiography (MRA) or X-ray contrast-agent based angiography, could be alternatives. Still, MRA has high costs and X-ray angiography causes exposure to radiation. Systolic toe pressures or toe-to-brachial pressure index (TBI) have also been suggested as an alternative to ABI especially to diabetic patients, but also they have problems with the reliability [6. Many studies have suggested pulse wave (PW) measurement and analysis for finding vascular abnormalities or abnormal vascular aging [7, 8, 9, 10, 11, 12].

Earlier, we have studied the repeatability of the PW-measurements and if there exist differences in the PW-morphologies between different-aged subjects as well as between healthy subjects and atherosclerotic patients in the signals recorded from various locations [11]. The experimental set-up of these studies limits the results only to provide information on the overall condition of the arterial tree without data on the effect of the stenosis itself. In this study, we test the suitability of the PW measurement and analysis for the monitoring of a superficial femoral artery (SFA) stenosis treated by PTA and provide information on the effects of PTA on PW. We hypothesize that PTA causes quantitative changes in the observed PW and that the effect of the treatment is observed immediately after the treatment and more clearly in the follow-up visit within one month due to remodeling initiated by the PTA. We also test if the studied PWanalysis methods are suitable for diagnostic use, e.g. if there are pre-treatment differences in the PW-features between the treated and non-treated lower limb. A benefit of the PW measurement compared with the static ABI measurement or angiographs is that it provides information also on the vascular dynamics.

\section{Materials and methods}

\subsection{Measurement hardware and sensor placement}

The measurement data was collected in supine position by using electret sensors made of electromechanical film (EMFi) (Emfit S-series, Emfit Ltd, Fin- 
land) and PPG probes (S0010A, Shenzhen Med-link, China) connected to a wireless body sensor network (WBSN) [13. The sampling frequency was 250 $\mathrm{Hz}$ for the EMFi signals and $500 \mathrm{~Hz}$ for the PPG signals. The EMFi signals were interpolated to have a sampling frequency of $500 \mathrm{~Hz}$ in further signal processing.

EMFi-sensors, which are sensitive only to dynamic but not to static loading, were placed at the wrist on the top of the radial artery, and both ankles on the top of the posterior tibial artery in order to record dynamic pressure PW signals, i.e. a signal proportional to the varying AC-component or pulse pressure of the blood pressure signal.

Transmission mode PPG probes having an excitation wavelength of $905 \mathrm{~nm}$ were placed on index finger and both 2nd toes for recording blood volume PW signals. In addition to the PW-signal, bipolar ECG (electrocardiogram) was also measured by using conventional silver-silver chloride $(\mathrm{Ag} / \mathrm{Cl})$ electrodes placed under the clavicles and an ECG-device compatible with the WBSN [13]. The ECG was utilized in the extraction of the PWs.

The measurements were conducted during the pre-, peri-, and post-treatment phases of the PTA and during the follow-up visit after one month (median 33 days, inter-quartile range (IQR) 30-36 days). For peri-procedural data collection, the sensors were placed before the normal preparation of the PTA and were removed within 5-10 minutes after the PTA. The sensor placement was similar in the follow-up visit, but the duration of the measurement was 5 minutes and was done before the ABI and toe pressure measurements.

The ankle PW-signals were excluded from the analysis since they were disturbed heavily especially during the treatment mainly for two reasons: First, the PAD patients commonly have extremely low-amplitude pressure PW-signals and therefore the measurement is also very sensitive to the correct positioning of the sensors. Second, the operating table in the PTA room was narrow, which often forced patient's lower limbs in such position that the structures containing EMFi sensors touched each other or the operating table, and this caused major artifacts to the signal. 


\subsection{Reference values}

The ABI is defined as a ratio of the systolic blood pressures measured by a cuff from an arm and an ankle, i.e. ABI $=P_{\text {ankle }} / P_{\text {brachial }}$. ABI was computed by dividing the highest ankle pressure (either ADP (arteria dorsalis pedis) or ATP (arteria tibialis posterior)) by the highest arm pressure (left or right). Toe pressures refer to the systolic pressures measured from the hallux. Both reference values were collected by using an automated measurement device, Falcon Pro (Viasonix, Israel). Pre-treatment (visit at polyclinics before the PTA) and follow-up visit ABI and toe pressure measurements were conducted as a part of normal clinical practice and used as the reference values in the study.

\subsection{Study subjects}

The inclusion criteria of the study were the abnormal ABI reading, i.e. $\mathrm{ABI}<0.9$ or $\mathrm{ABI}>1.3$, relevant symptoms, stenosis in SFA based on magnetic resonance imaging angiography and a patient considered as a candidate for the PTA of the SFA. A pacemaker and a possible risk that the study interferes the patient's treatment process were considered as exclusion criteria. Altogether 27 volunteer patients undergoing PTA of the SFA were recruited for the study. Three of them were excluded for the following reasons: research personnel unavailable, a patient was found to be unsuitable for PTA during

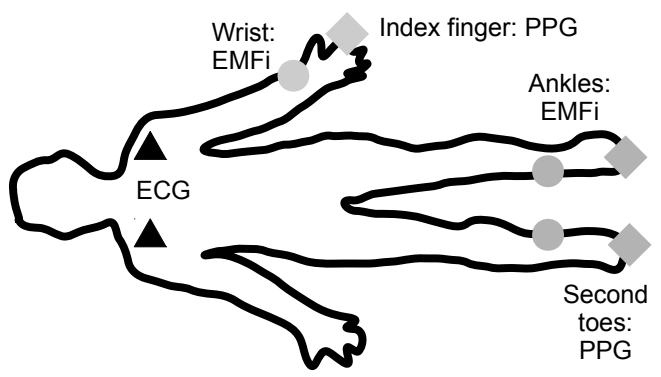

Figure 1: Sensor placement. 
the imaging and extremely low peripheral perfusion. 14 left and 12 right lower limbs were treated, including two patients with both lower limbs treated. More detailed data on the study subjects is shown in Table1. A clear majority of the included patients met the criterion $\mathrm{ABI}<0.9$ - only one of the patients had pre-treatment ABI higher than 1.3. 15 non-treated lower limbs out of 22 lower limbs $(68 \%)$ also had the ABI value outside the normal range of $0.9<\mathrm{ABI}<1.3$ at least in one of the pre-treatment or follow-up visit ABI measurements.

Table 1: Data describing the study subject population including clinical reference values, ABI and toe pressure.

\begin{tabular}{lc}
\hline \hline Parameter & Median (IQR) \\
\hline Mass $(\mathrm{kg})$ & $75.5(67.0 \ldots 93.0)$ \\
Height $(\mathrm{cm})$ & $170.0(165.5 \ldots 176.0)$ \\
BMI $\left(\mathrm{kg} / \mathrm{m}^{2}\right)$ & $26.7(24.4 \ldots 30.0)$ \\
Age $($ years $)$ & $71.5(67.5 \ldots 76.0)$ \\
\hline Treated lower limbs: 26 & \\
\hline ABI, pre-treatment & $0.61(0.50 \ldots . .75) *$ \\
ABI, follow-up & $0.96(0.82 \ldots 1.04) \#$ \\
Toe pressure, pre-treatment $(\mathrm{mmHg})$ & $59.0(42.0 \ldots 86.0)^{* *}$ \\
Toe pressure, follow-up $(\mathrm{mmHg})$ & $94.5(76.0 \ldots 122.0) \# \#$ \\
\hline Non-treated lower limbs: 22 & \\
\hline ABI, pre-treatment & $0.88(0.75 \ldots 1.02) *$ \\
ABI, follow-up & $0.94(0.72 \ldots 1.08) \#$ \\
Toe pressure, pre-treatment $(\mathrm{mmHg})$ & $75.5(53.0 \ldots 117.5) * *$ \\
Toe pressure, follow-up $(\mathrm{mmHg})$ & $99.5(66.0 \ldots 122.0) \# \#$ \\
\hline Parameter & Number $(\%)$ \\
\hline Males & $16(66.7 \%)$ \\
Diabetes & $11(45.8 \%)$ \\
Dyslipidemia & $22(91.7 \%)$ \\
Rheumatoid arthritis & $3(12.5 \%)$ \\
Current smoker & $2(8.3 \%)$ \\
Ex-smoker & $11(45.8 \%)$ \\
\hline
\end{tabular}

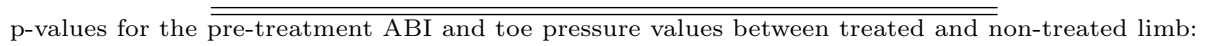
$*: p<0.001, * *: p<0.1, \#$ and \#\#: not signigicant BMI: Body-mass index; IQR: inter-quartile range.

\subsection{Ethics and patient safety}

The study was approved by the local ethical review board of the hospital district (R15107), the Finnish National Supervisory Authority of Health and 
Welfare (Valvira, ID 309) and the technical department of the hospital. The study was registered to a public clinical trials register (ClinicalTrials.gov ID: NCT02725307). Written informed consents were obtained from the volunteer patients participating in the study.

\subsection{Pulse wave analysis}

The PW-extraction was implemented first by finding R-peaks from the ECG by an algorithm proposed in [14]. After that, local mimima following each detected R-peak were extracted from the pulse wave signals. The pulse wave signal between the successive local minima was considered as a PW candidate. The validity of the PW candidates was tested by using adaptive thresholds. A PW candidate was rejected if 1) the slope of the rising edge of the PW candidate was not positive for $50 \mathrm{~ms}$ or if its average slope was less than $30 \%$ of the maximum slope; 2) the difference of the minimum and maximum value of the PW-candidate was outside certain thresholds; or 3) amplitude-normalized PW candidate was outside predefined limits for at least $20 \%$ of its duration.

In pre-processing, the signals were filtered with a Savitzky-Golay smoothing filter having a window length of 91 samples (182 ms) and a polynomial order of

2. In addition, the signals were forward-backward filtered with a finite impulse response low-pass filter having a cutoff frequency of $10 \mathrm{~Hz}$, transition band of 10-12 Hz, pass band ripple of $0.05 \mathrm{~dB}$, and stop band attenuation of $100 \mathrm{~dB}$ [15], as implemented in [16, 11]. After pre-processing and feature extraction, the PWs were characterized by computing altogether 10 different PW-parameters.

\subsection{1. $P W$-curve derived features}

The features extracted from the PWs were originally proposed either for radial pressure $\mathrm{PW}$-analysis or digital volume (PPG) $\mathrm{PW}$-analysis as e.g. in [17, 18. In earlier studies [16, 11, we extracted similar features also from the lower limb PWs by using algorithms that were originally implemented for upper limb PWs and proposed in [15, 19. In this study, we analyzed how these features, extracted from lower limb PWs, are changed as a result of the PTA of 
the SFA. Numerous studies have presented results that the morphology of both pressure and volume pulse wave depends on the status of the vasculature. The resistance and compliance of the arterial pathway affects the wave propagation and reflection [9], but the exact physiological origin and significance of the differences requires further studies especially in case of lower limbs.

Different parameters were computed based on the amplitudes and time differences between the systolic and diastolic waves as in [1] and illustrated in Fig. 2 When finding the fiducial points for $P_{1}, P_{2}$, and $B$, the incisura or dicrotic notch dividing the individual PW into systolic and diastolic parts was found as the last zero-crossing from negative to positive of the $1^{\text {st }}$ derivative $f^{\prime}$ of the $\mathrm{PW}$ in the search window limited by a point $80 \mathrm{~ms}$ after the maximum of the PW and a point which corresponds to $65 \%$ from the total length of the PW. In case of the absence of this zero-crossing, the location of incisura was detected at the location of the highest peak of the $2^{\text {nd }}$ derivative $f^{\prime \prime}$ found from the same interval [16. Based on these features, the following parameters were computed:

- $R_{1}$ as a ratio of the amplitude of diastolic wave $B$ and the systolic maximum (the maximum of $P_{1}$ and $P_{2}$ in Fig. 2 , i.e. $R_{1}=B / \max \left(P_{1}, P_{2}\right)$, named as reflection index for index-finger PPG in [18,

- $R_{2}$ as a ratio of the amplitude of diastolic wave $B$ and early systolic wave $P_{1}$, i.e. $R_{2}=B / P_{1}$

- $R_{3}$ as a ratio of the amplitude of diastolic wave $B$ and late systolic wave $P_{2}$, i.e. $R_{3}=B / P_{2}$

- $R_{4}$ as a ratio of the amplitude of late $\left(P_{2}\right)$ and early $\left(P_{1}\right)$ systolic wave, i.e. $R_{4}=P_{2} / P_{1}$, named as peripheral augmentation index for wrist pressure pulses e.g. in [17,

- $T_{1}$ as a time difference between systolic maximum $\left(\max \left(P_{1}, P_{2}\right)\right)$ and the peak of the diastolic wave $(B)$ 


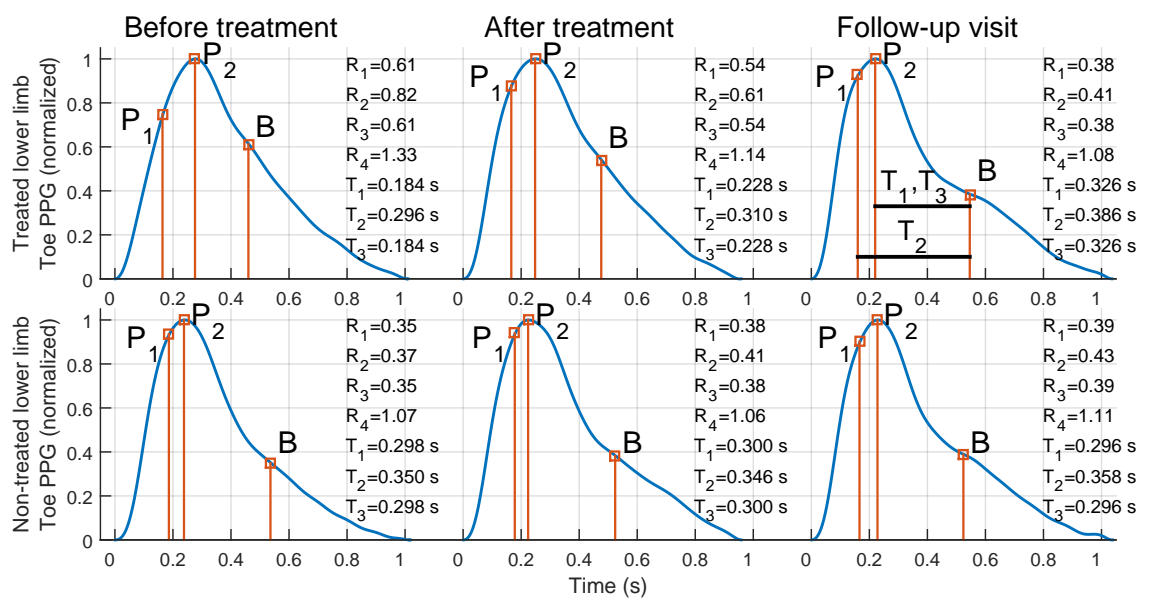

Figure 2: Examples of the PWs, locations of fiducial points and time intervals, and values of the derived parameters from one test subject before and after the PTA as well as during the follow-up visit. Also $T_{1}-T_{3}$ are illustrated in the PW shown in the right upper corner. $T_{1}$ and $T_{3}$ are equal in all these examples, since $P_{2}$ is the maximum of the $\mathrm{PW}$ in all the examples. Each pair of the PWs from treated and non-treated lower limb are recorded concomitantly.

- $T_{2}$ as a time difference between early systolic peak $\left(P_{1}\right)$ and the diastolic peak $(B)$

- $T_{3}$ as a time difference between late systolic peak $\left(P_{2}\right)$ and the diastolic peak $(B)$

The peaks of early and late systolic waves $P_{1}$ (Fig. 2) and $P_{2}$ as well as diastolic wave $B$ were found based on the method described in [16, 19. These peaks are often overlapped in the PWs recorded from atherosclerotic patients, but they are more obvious in radial or carotid PWs and in lower limb PWs recorded from healthy subjects [16, 11. For these reasons, the fiducial points were found by implementing $5^{\text {th }}$-order derivative analysis as in [16, 19].

After finding the boundary between the systolic and diastolic parts, the following procedure was implemented for finding $P_{1}$ and $P_{2}$ [16, 19]: If the sign of the $5^{\text {th }}$ derivative $f^{(5)}$ at the point corresponding to the systolic maximum of the PW was

1. positive, this point was considered as a point for the late systolic peak $P_{2}$ 
and the last zero-crossing of $4^{\text {th }}$ derivative $f^{(4)}$ from positive to negative before $P_{2}$ as early systolic peak $P_{1}$.

2. negative, this point was considered as a point for the early systolic peak $P_{1}$ and the first zero-crossing of $f^{(4)}$ from negative to positive after $P_{1}$ as a late systolic peak $P_{2}$.

In order to find the diastolic wave $B$, the zero-crossing of $1^{\text {st }}$ derivative $f^{\prime}$ from positive to negative in the diastolic part of the $\mathrm{PW}$ was selected as the location of the diastolic peak. If such diastolic zero-crossing of $f^{\prime}$ was missing, the location for the diastolic peak was defined as the first zero-crossing of $2^{\text {nd }}$ derivative $f^{\prime \prime}$ from positive to negative in the diastolic part of the PW [16].

Aging index (AGI) based on the 2nd derivative of an individual toe-PPG $\mathrm{PW}$ signal was computed as AGI $=(b-c-d-e) / a$ in which $a$ is the peak amplitude of the 2nd derivative of the $\mathrm{PW}$ and $b, c, d$, and $e$ are the amplitudes of the following local extremities of the 2 nd derivate [18].

The amplitude ratios $R_{1}-R_{4}$ and AGI derived for the lower limb PPG are observed to increase with age and atherosclerotic changes and time delays $T_{1}-T_{3}$ to decrease with age and atherosclerotic changes in [11, 16].

\subsubsection{Area ratios}

Two different ratios of areas under amplitude-normalized PWs recorded from different locations were extracted as

- $A_{\mathrm{wt}}=A_{\mathrm{wrist}} / A_{\mathrm{toe}}$

- $A_{\mathrm{ft}}=A_{\text {finger }} / A_{\text {toe }}$

in which $A_{\text {wrist }}, A_{\text {toe }}$ and $A_{\text {finger }}$ refer to area under wrist, toe, and finger PWs, respectively. These areas were computed by using trapezoidal numerical integration.

\subsection{Statistical methods}

The distributions of the values of the PW-features found for the treated and non-treated lower limb were compared by implementing non-parametric two- 
tailed Mann-Whitney U-tests because of relatively small sample sizes and nonsymmetric distributions. The pair-wise differences between the values before and after the PTA as well as in the follow-up visit were compared by implementing two-tailed non-parametric sign tests for the same reasons as with the Mann-Whitney U-tests.

Also the peri-procedural changes in the values of the $\mathrm{PW}$-features caused by the inserted endovascular instrumentation were analyzed by means of sign tests in two cases: 1) before any endovascular preparation vs. all the necessary equipment inserted to the SFA, and 2) immediately after the balloon angioplasty or a possible stent installation vs. all the endovascular instruments removed. The non-parametric methods instead of parametric tests were selected because of the relatively small sample sizes and skewness of the distributions of the part of the parameters.

Bland-Altman (BA) plot analysis was implemented for both PW-derived indices as well as reference values in order to see if there are differences between the groups of treated and non-treated lower limbs. In the BA-plot, the difference between two measurements ( $\left.p_{\text {follow }- \text { up }}-p_{\text {before }}\right)$ is plotted against the average of two measurements $\left(1 / 2 \cdot\left(p_{\text {before }}+p_{\text {follow-up }}\right)\right)$ in which $p_{\text {before }}$ is the pretreatment value and $p_{\text {follow-up }}$ is the value in the follow-up visit.

In order to study if the values of the PW-features and reference values (ABI and toe pressure) change in the same direction as a result of treatment, fixedmarginal multirater $\kappa$-analysis 20 was performed for the values found for the treated lower limbs. If the value develops in a healthier direction, i.e. the waveform changes towards the values measured for healthy volunteers in [1] as a result of the treatment, that value was categorized as positive change, and if the value develops towards worse condition, that value was categorized as negative change. The $\kappa$-analysis is performed between $\mathrm{PW}$-derived indices and ABI as well as between PW-derived indices and toe pressures. An increase in $\mathrm{ABI}$ and toe pressure value is considered as a positive change towards healthier condition.

Multiple linear regression models are constructed for finding if the change 
in the values is explained by the treatment and remodeling or other changing factors.

\subsection{Pre-treatment repeatability}

Pre-treatment beat-to-beat repetability was studied by means of the intraclass correlation coefficient (ICC) based on the one-way random effect model [21, 22] and coefficient of variation (CV). ICC was used to estimate the repeatability of the series of measurements and it was defined as a ratio of betweensubject variance and the sum of between-subject and within-subject variances and estimated as

$$
\mathrm{ICC}=\frac{\mathrm{MS}_{\mathrm{bs}}-\mathrm{MS}_{\mathrm{ws}}}{\mathrm{MS}_{\mathrm{bs}}+(k-1) \mathrm{MS}_{\mathrm{ws}}}
$$

in which $\mathrm{MS}_{\mathrm{bs}}$ is between-subject mean squares, $\mathrm{MS}_{\mathrm{ws}}$ is within-subject mean squares from ANOVA (analysis of variance) table and $k$ is the number of observations per subject which computed as in 22] in case of unequal number of

observation per test subject as in this case. CV is defined as a ratio between sample standard deviation $s$ and mean $m$ as $\mathrm{CV}=s / m$.

\section{Results}

The amplitude ratio $R_{4}$ and AGI did not reveal any statistically significant differences between any of the studied situations or between treated and nontreated limbs, so more detailed results related to them are not reported.

\subsection{Distributions of the $P W$-parameters between pre-treatment state and follow- up visit}

The distributions of the values of the PW-features before the treatment and at the follow-up visit are presented in Table 2 in terms of median and IQR for both treated and non-treated lower limbs. Generally, there were no differences at group level in the pre-treatment values between treated and non-treated limbs. The area ratio $A_{\mathrm{ft}}$ was an exception $(p<0.05)$ suggesting only weak difference between the pre-treatment condition of treated and non-treated limb. If the 
Table 2: Distributions (median and IQR) of the values of the PW-features for treated and non-treated limb for both pre-treatment condition and a situation at the follow-up visit.

\begin{tabular}{|c|c|c|c|c|}
\hline \multirow{3}{*}{$\begin{array}{l}\text { Para- } \\
\text { meter }\end{array}$} & \multicolumn{2}{|c|}{ Pre-treatment } & \multicolumn{2}{|c|}{ Follow-up visit } \\
\hline & Treated limb & Non-treated limb & Treated limb & Non-treated limb \\
\hline & Median (IQR) & Median (IQR) & Median (IQR) & Median (IQR) \\
\hline$R_{1}$ & $0.52(0.45 \ldots 0.57)$ & $0.47(0.32 \ldots 0.53)$ & $0.34(0.27 \ldots 0.44)$ & $0.38(0.28 \ldots 0.53)$ \\
\hline$R_{2}$ & $0.56(0.48 \ldots 0.63)$ & $0.52(0.34 \ldots 0.62)$ & $0.37(0.29 \ldots 0.50)$ & $0.40(0.29 \ldots 0.54)$ \\
\hline$R_{3}$ & $0.56(0.50 \ldots 0.61)$ & $0.51(0.33 \ldots 0.60)$ & $0.40(0.27 \ldots 0.49)$ & $0.43(0.28 \ldots 0.58)$ \\
\hline$T_{1}$ & $0.27(0.23 \ldots 0.36)$ & $0.30(0.24 \ldots 0.35)$ & $0.34(0.31 \ldots 0.39)$ & $0.31(0.26 \ldots 0.35)$ \\
\hline$T_{2}$ & $0.31(0.28 \ldots 0.40)$ & $0.34(0.28 \ldots 0.38)$ & $0.36(0.34 \ldots 0.43)$ & $0.35(0.30 \ldots 0.42)$ \\
\hline$T_{3}$ & $0.22(0.19 \ldots 0.30)$ & $0.25(0.21 \ldots 0.32)$ & $0.30(0.25 \ldots 0.36)$ & $0.29(0.23 \ldots 0.35)$ \\
\hline$A_{w t}$ & $0.81(0.78 \ldots 0.89)$ & $0.86(0.82 \ldots 0.94)$ & $0.90(0.84 \ldots 0.97)$ & $0.87(0.80 \ldots 0.99)$ \\
\hline$A_{f t}$ & $0.91(0.87 \ldots 0.98) \bullet$ & $0.99(0.91 \ldots 1.07) \bullet$ & $0.97(0.90 \ldots 1.11)$ & $0.97(0.86 \ldots 1.08)$ \\
\hline
\end{tabular}

pre-treatment reference values are studied, ABI provides statistically significant differences between treated and non-treated lower limb whereas the sample size is slightly too small for finding significant differences with toe pressures (Table 1). Neither the PW-derived parameters (Table 2 ) nor the reference values (Table 1) show differences between the distributions of treated and non-treated lower limbs at the follow-up visit.

\subsection{Changes during the PTA}

Clear changes during the PTA were observed only when the PTA-balloon was filled: at those points, the amplitude of the PW decreased at least $50 \%$ except for one test subject. According to the implemented statistical tests, the inserted endovascular instrumentation does not otherwise cause changes to the values of studied PW-parameters.

\subsection{Immediate pre- and post-treatment changes}

The immediate changes caused by the treatment are relatively small (Figs. $3 \mathrm{a}-\mathrm{c}$ ) compared with changes found in the follow-up visit (Figs. $3 \mathrm{~h}-\mathrm{j}$ ). Statistically significant differences for these immediate changes in the values of the $\mathrm{PW}$-features of the treated limbs were found only for $R_{2}, T_{2}, T_{3}$, and $A_{\mathrm{ft}}$ even 

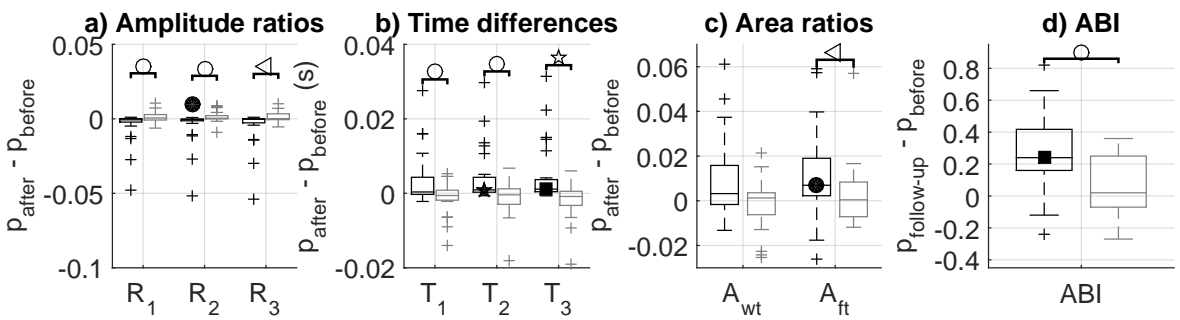

$\begin{array}{ll}\text { e) Amplitude ratios } & \text { f) Time differences }\end{array}$
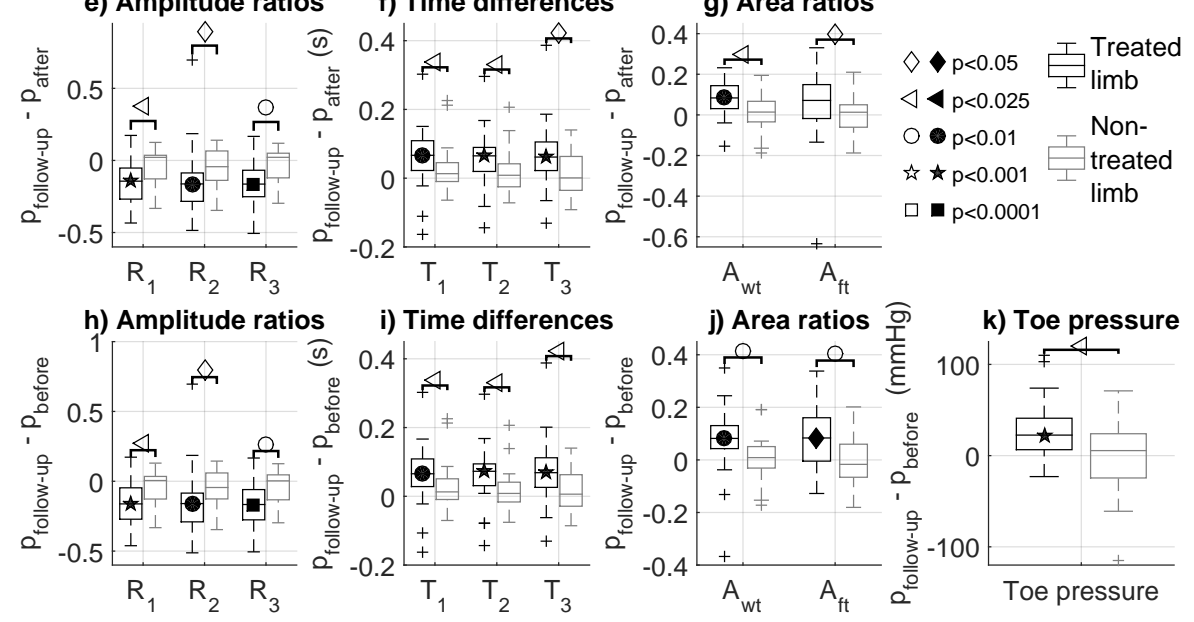

Figure 3: Distributions for the changes of different values: in each pair of boxplots, the blackbordered left-hand side box is for the treated lower limbs and the gray-bordered right-hand side box is for the non-treated lower limbs. In the vertical axis, $p_{\text {after }}$ refers to the posttreatment values immediately after the treatment, $p_{\text {before }}$ to the values immediately before the treatment and $p_{\text {follow-up }}$ to the values in the follow-up visit.

though the trend of other values is also in the healthier direction as described in [11.

The changes found for the values of the PW-features of the non-treated limb do not show any statistically significant differences between pre-treatment and post-treatment conditions. With most of the parameters $\left(R_{1}-R_{3}, T_{1}-T_{3}\right.$, and $\left.A_{\mathrm{ft}}\right)$ the distribution of the immediate changes in the values of the treated limbs differ significantly from changes found for the non-treated limbs (Figs. 3 a $-\mathrm{c}$ ). 


\subsection{Changes in the follow-up visit}

The changes in amplitude ratios $R_{1}-R_{3}$, time differences $T_{1}-T_{3}$ and area ratio $A_{\mathrm{wt}}$ for the treated lower limb indicate statistically significant differences between the follow-up visit and the post-treatment situation as well as between follow-up visit and pre-treatment situation (Figs. 3e-j). With these parameters, the distributions of the changes found for the treated and non-treated limb from differ each other statistically, and this is also observed by a visual inspection: the changes in the non-treated limb are clearly more zero-centered than the changes in the treated limb in which the trend is in the healthier direction reported in [16. These trends in the amplitude ratios $\left(R_{i}\right)$ and time differences $\left(T_{i}\right)$ are also seen in the examples of the PWs recorded in different situations shown in Fig. 2.

\subsection{Comparison with $A B I$ and toe pressures}

The BA-plots are shown in Fig. 4 for the pre-treatment situation and the follow-up visit for the PW-derived parameters as well as for the reference values ABI and toe pressures. The $95 \%$ limits of agreement were not drawn for the treated limbs since the change in the measurand as a result of the PTA is assumed. In the ideal case and after successful PTA treatment, the BA-plot should indicate no difference or deviation in the readings of the non-treated limb and a consistent difference (bias) towards healthier condition in the treated limb. With the PW-parameters, the deviations are high for both treated and non-treated limbs. However, this is the case also for the reference values: For treated limbs, bias and $95 \%$ limits of agreement of $0.27 \pm 0.47$ and $(28.9 \pm 64.5)$ $\mathrm{mmHg}$ were found for the ABI and toe pressure, respectively. Even for the nontreated limb, bias and $95 \%$ limits of agreement of $0.06 \pm 0.37$ and $(0.2 \pm 82.0)$ $\mathrm{mmHg}$ were found for $\mathrm{ABI}$ and toe pressure, respectively.

In addition to BA-plots, the free-marginal multirater $\kappa$-values between PWderived parameters and the reference values, ABI and toe pressures, of the treated lower limb are shown in Table 3 . All the PW-derived parameters which showed statistically significant differences between the pre-treatment situation 
and follow-up visit have at least moderate acceptance with the ABI, and the $\kappa$-values between $\mathrm{ABI}$ and $R_{1}, R_{3}, T_{1}$ and $T_{2}$ parameters are even higher than the $\kappa$-value between ABI and toe pressure (0.75). All $\kappa$-values between the ABI and PW-derived parameters are higher than corresponding values between toe pressures and $\mathrm{PW}$-derived parameters.

Table 3: Free-marginal multirater $\kappa$ values for the changes between different values and ABI and toe pressure.

\begin{tabular}{llc}
\hline \hline Parameter & ABI & Toe pressure \\
\hline$R_{1}$ & 0.82 & 0.62 \\
$R_{2}$ & 0.73 & 0.52 \\
$R_{3}$ & 0.91 & 0.71 \\
\hline$T_{1}$ & 0.73 & 0.62 \\
$T_{2}$ & 0.82 & 0.71 \\
$T_{3}$ & 0.82 & 0.71 \\
\hline$A_{\mathrm{wt}}$ & 0.52 & 0.30 \\
$A_{\mathrm{ft}}$ & 0.55 & 0.52 \\
\hline Toe pressure & 0.75 & \\
\hline \hline
\end{tabular}

\subsection{Multiple linear regression}

The results of multiple linear regression analysis for the changes between pre-treatment situation and follow-up visit are shown in Table 4. According to the results, the changes are dependent on the treatment of the SFA but not on the RR-interval except $T_{2}$. Surprizingly the toe pressures are less dependent on the treatment of the SFA than PW-derived indices and ABI.

\subsection{Pre-treatment repeatability}

Both ICC and CV are shown in Table 5 for beat-to-beat data in pre-treatment situation. ICCs close to 0.9 or higher and median CVs less or equal than $2 \%$ were observed. Both indicators show good repeatability and are inline with the earlier study [11]. 


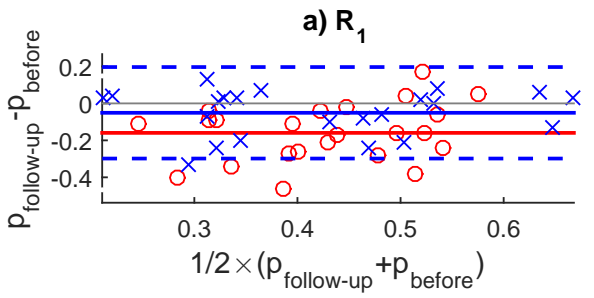

c) $\mathbf{R}_{3}$

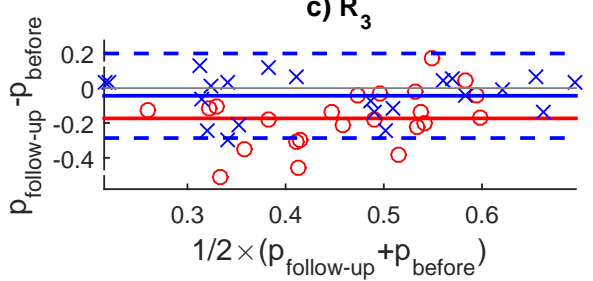

e) Finger/Toe $\left(A_{\mathrm{ft}}\right)$

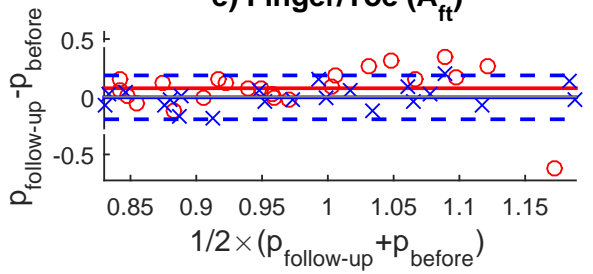

(5)

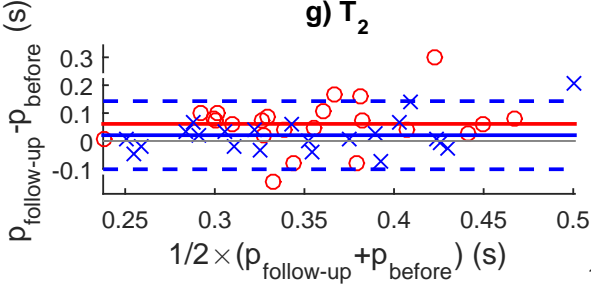

i) Reference value: $A B$
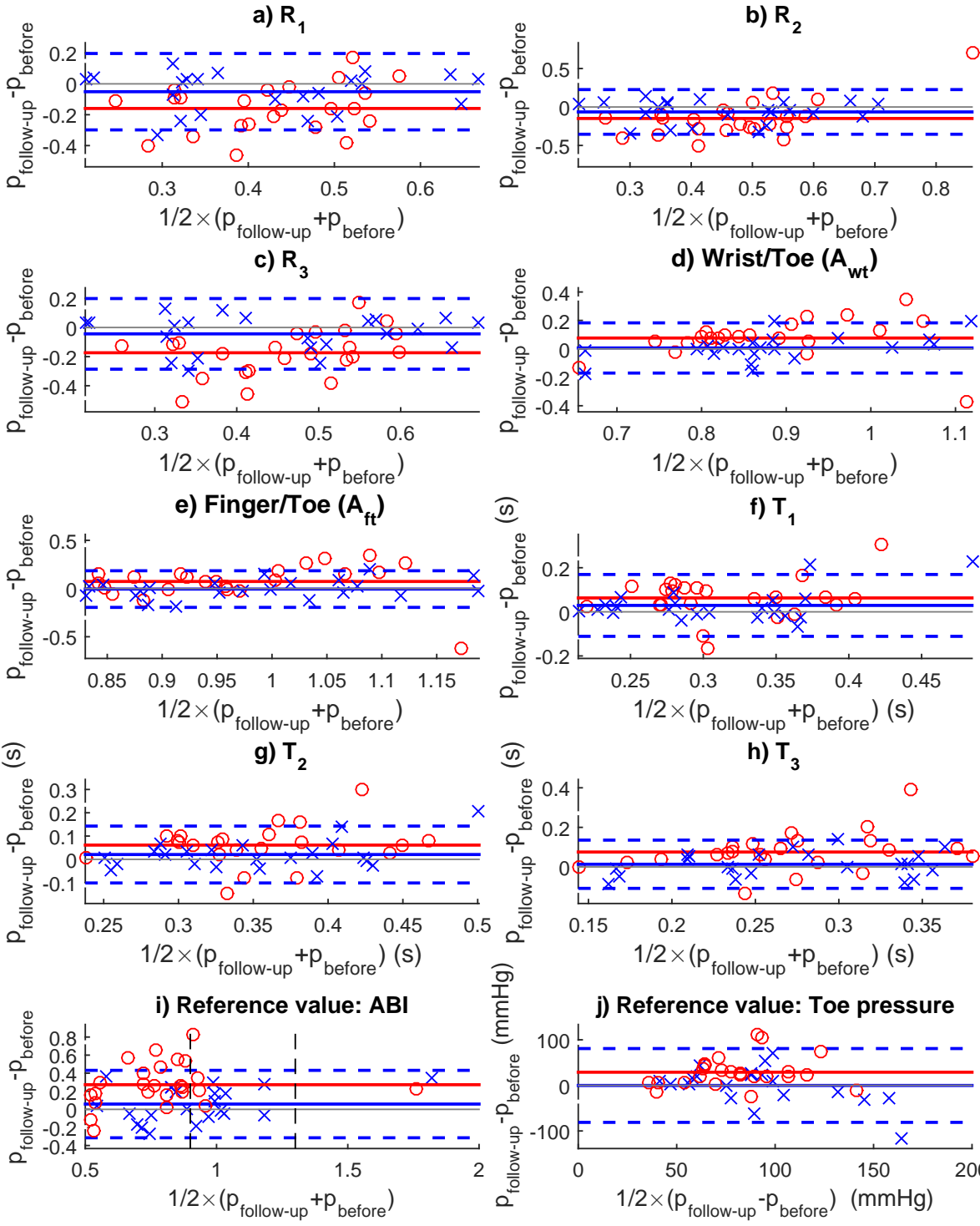

d) Wrist/Toe $\left(A_{w t}\right)$

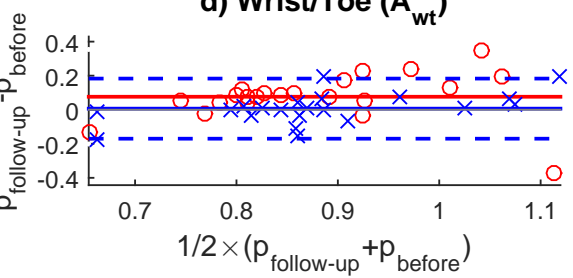

क

f) $T_{1}$

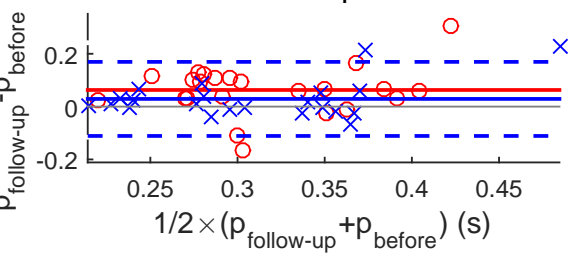

()

h) $T_{3}$
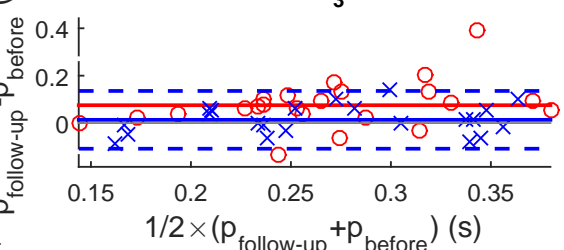

오 ह

j) Reference value: Toe pressure

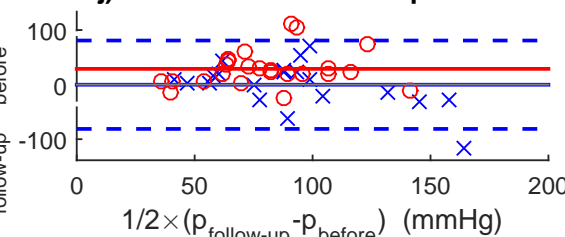

$\times \quad$ Non-operated limb

- Average difference (non-operated limb)

- - $-95 \%$ limits of agreement (non-operated limb)

O Operated limb

Average difference (operated limb)

Figure 4: Bland-Altman plots for the pre-treatment values and the values found in the followup visit for each $\mathrm{PW}$-parameter and the reference values ABI and toe pressure. The normal range for $\mathrm{ABI}$ is also shown in black vertical dashed lines in panel i). 
Table 4: Multiple linear regression coefficients and significance levels. The marker after parameter name refers to p-value of the F-statistics vs. constant model.

\begin{tabular}{c|ccc}
\hline \hline & \multicolumn{3}{|c}{ Coefficients } \\
Parameter & Intercept & $\Delta$ RR & SFA PTA \\
\hline$R_{1} \circ$ & -0.047 & -0.164 & $-0.116 \bullet$ \\
$R_{2} \bullet$ & -0.058 & -0.257 & $-0.141 \bullet$ \\
$R_{3} \bullet$ & -0.041 & -0.134 & $-0.133 \triangleleft$ \\
\hline$T_{1} \bullet$ & 0.013 & -0.056 & $0.054 \triangleleft$ \\
$T_{2} \triangleleft$ & 0.010 & $-0.235 \bullet$ & $0.047 \bullet$ \\
$T_{3} \circ$ & 0.011 & -0.129 & $0.052 \bullet$ \\
\hline$A_{\mathrm{wt}} \bullet$ & 0.005 & 0.047 & $0.086 \triangleleft$ \\
$A_{\mathrm{ft}} \bullet$ & -0.004 & 0.123 & $0.108 \triangleleft$ \\
\hline $\mathrm{ABI} \triangleleft$ & -0.053 & 0.490 & $-0.210 \triangleleft$ \\
Toe pressure & -4.731 & 19.928 & $-20.727^{*}$ \\
\hline \hline
\end{tabular}

$\Delta$ RR: change in RR-interval
$*: p<0.10, \circ: p<0.05, \bullet: p<0.025, \triangleleft: p<0.01$

Table 5: Intra-class correlations (ICC) and coefficients of variation (CV) for pre-treatment measurement.

\begin{tabular}{c|cc}
\hline \hline Parameter & ICC $(95 \%$ confidence limit) & Median CV (1st...3rd quartile) $(\%)$ \\
\hline$R_{1}$ & $0.96(0.93-0.97)$ & $0.6(0.4 \ldots 3.0)$ \\
$R_{2}$ & $0.96(0.93-0.97)$ & $0.5(0.3 \ldots 3.0)$ \\
$R_{3}$ & $0.96(0.94-0.97)$ & $0.6(0.4 \ldots 2.9)$ \\
\hline$T_{1}$ & $0.89(0.84-0.93)$ & $1.5(0.9 \ldots 2.7)$ \\
$T_{2}$ & $0.89(0.83-0.92)$ & $1.5(0.8 \ldots 3.5)$ \\
$T_{3}$ & $0.91(0.87-0.94)$ & $2.0(1.2 \ldots 4.0)$ \\
\hline$A_{w t}$ & $0.96(0.94-0.97)$ & $1.9(1.4 \ldots 3.2)$ \\
$A_{f t}$ & $0.97(0.96-0.98)$ & $1.3(1.0 \ldots 1.8)$ \\
\hline \hline
\end{tabular}

\section{Discussion}

The presented results suggest that PTA caused significant changes in the features extracted from the PWs. The majority $\left(R_{1}-R_{3}, T_{1}-T_{3}, A_{\mathrm{wt}}\right.$, and $\left.A_{\mathrm{ft}}\right)$ of the studied parameters showed statistically significant differences between pretreatment condition and a situation at the follow-up visit. Only $R_{2}, T_{2}-T_{3}$, and $A_{\mathrm{ft}}$ showed differences between the pre-treatment and immediate post-treatment values, but other PW-derived parameters still had trends towards healthier condition. The PW-measurement is able to find some immediate changes, but the results suggest that the remodeling of the plaque and arterial wall within the 
follow-up period makes the changes more visible. Therefore, the behaviour of the post-treatment $\mathrm{PW}$-parameters is similar to the post-treatment $\mathrm{ABI}$ as proposed in 23] and especially if the pre-treatment ABI is less than 0.80 [24].

According to the results, the endovascular instrumentation inserted into SFA does not affect the values of the peri-procedural PW-parameters. The independence of the PW-parameters on the insertion of the endovascular instruments could allow to monitor changes in PW morphology caused by the PTA in real time, even though the immediate changes could be small.

\subsection{PW-analysis and changes caused by PAD}

To the extent of our knowledge, the presented study is the first study which compares the values of lower-limb PW-derived parameters between the pre- and post-treatment situations of the PTA of the SFA. However, the results are supported by other studies concentrating on the diagnosis of the PAD: Yokoyama et al. 25. have reported an increase in PW velocity in diabetic patients due to arterial stiffness, but decrease in the PW velocity in a lower limb having PAD symptoms likely due to stenoses which decrease the blood pressure and thus the PW velocity in the diseased lower limb. After the successful PTA, the PW velocity increased indicating the changes in the lower limb vasculature. Similar kind of results were found in this study: the values of the PW-derived parameters change in the healthier direction as reported earlier in [11.

The PW-analysis can also reveal the beneficial wide-scale systemic remote effects of the PTA: Jacomella et al. [12] reported changes towards better condition within 3-month follow-up period of the PTA of the SFA in a carotid artery augmentation index based on a radial artery applanation tonometry. In the present study, the medians of the distributions of $R_{1}-R_{3}$ in non-treated limbs (Table 22) shifted towards healthier values observed in [1] during the 1-month followup period even though the patient-wise differences between pre-treatment and follow-up visit situations were close to zero (Figs. $3 \mathrm{~h}-\mathrm{j}$ ). However, the changes in the IQRs were not as dramatic in Table 2. We assume that a successful PTA restores physical activity, such as walking, also improving the condition of the 
contralateral lower limb and resulting in increased perfusion in the lower limbs. Similar changes were also observed in the clinical references values (Table 1 ) and Figs. $3 \mathrm{~d}$ and $3 \mathrm{k}$ ) which supports the assumption. The area ratios $A_{\mathrm{wt}}$ and $A_{\mathrm{ft}}$ as well as time intervals $T_{1}-T_{3}$ behave differently: their distributions at pre-PTA and follow-up visit situations did not differ from each other in case of non-operated lower limbs (Table 2), indicating weaker dependence on positive systemic remote effects. Further studies should confirm these assumptions.

There are also studies [7, 8, 9, 10, reporting bilateral differences between the lower limbs with and without PAD. Erts et al. [10] have reported time differences in the foot points (the beginnings of the PW) between the lower limb with and without the PAD. Allen et al. [7, 8, 9] have found bilateral differences between toe-PPG signals in unilateral PAD patients. A comparison between different time delays between the peak or foot points of the PWs from different locations and between the patients and control subjects is also in our future interests.

\subsection{Limitations of the method}

In previous study[11], statistically significant differences were found for the study groups consisting of patients having atherosclerotic changes and sameaged healthy control subjects. The experimental setup in the previous study [11 was different than in this study in which the lower limbs that underwent SFA of the PTA were compared with the lower limbs without the PTA. The results of the present study do not show significant bilateral differences between the treated limb and the non-treatment limb if the pre-treatment values of the PW-derived parameters are studied. This possibly limits the diagnostic use of the studied PW-analysis methods.

Possible reason is that $68 \%$ of non-treated lower limbs provided abnormal $\mathrm{ABI}$ reading at least in one of the two measurements. If a patient has the symptoms of PAD in one limb, there is a high likelihood of atherosclerotic changes also contralaterally and elsewhere in the vasculature. The studied PW-derived parameters may also be sensitive to all kinds of degenerative atherosclerotic changes including the stiffening of the large arteries such as the aorta, not only 
the stenoses and occlusions which are the typical lesions of the PAD. Still, depending on the method, either bilateral comparison or unilateral measurement can provide the better performance, even though the differences are small as in [8, 9.

If the obvious sources of error in the PPG-measurement, such as incorrectly placed PPG-probe and motion artefacts, are excluded, there are a few methodological limitations. Poor peripheral perfusion decreases the amplitude of the PPG-signal and may prevent the proposed analysis. The low-amplitude PPGsignal can be a marker of PAD, but can also be caused by vasoconstriction as the result of low temperature. The body position affects the shape of the PPG-signal and is a topic of further studies. However, in the present study, all the measurements were conducted in supine position and limbs extented. Light from the environment can disturb the PPG-signals, but in our measurements, regular $50 \mathrm{~Hz}$ and $100 \mathrm{~Hz}$ frequency components caused by the mains and the fluorescent lights were the most significant sources of disturbance and were eliminated by the filtering.

Despite the limitations discussed, the results suggest that PTA has an effect on most of the features extracted and evaluated in the present study. The analysis of the PW-features may provide additional information in finding the early signs of $\mathrm{PAD}$ or in monitoring the treatment during the follow-up period especially in the cases in which the conventional methods, ABI and toe pressure measurements, provide in unreliable or conflicting results. A part of the features combined with other health data may be utilized also as the inputs of

multivariate analysis methods or machine learning which would be a topic of further studies.

\section{Conclusions}

Statistically significant differences were found in the values of the PWderived features between pre-treatment and immediate post-treatment condition. Stronger differences in the treated lower limb were found between the 
pre-treatment values and the post-treatment conditions after a 1-month followup period. In both cases, the changes in the values of the treated limb differed significantly from the corresponding changes found for the non-treated lower limb. In case of the non-treated lower limbs, the variations of the differences between different measurement events were relatively high but without a consistent trend towards healthier or worse condition. The endovascular instrumentation inserted into SFA did not affect the PW-parameters. The study was not able to show statistically significant differences in the pre-treatment values of the PW-derived parameters between the treated and non-treated lower limbs. This is possibly explained by the study sample: $68 \%$ of the non-treated limbs produced an abnormal ABI-reading at least for one of the two measurements. This indicates that many patients had subclinical atherosclerotic changes also in the other lower limb. Prior to the real utilization of the measurement method, further comparison of the healthy subjects and PAD-patients and having standardized measurement conditions should confirm the findings.

\section{Conflict of interest statement}

The authors declare no conflict of interest.

\section{Acknowledgment}

We would like to thank all the volunteer test subjects for their valuable contribution. The personnel in Tampere University Hospital (Department of Vascular Surgery) are acknowledged for their contribution to collecting the reference values. The work was funded by the Doctoral Programme of the President of Tampere University of Technology, Finnish Funding Agency for Innovation (TEKES) as a part of project VitalSens (decision ID 40103/14), and grants from Finnish Cultural Foundation/Pirkanmaa Regional Fund/Elli and Elvi Oksanen's Fund, Tekniikan edistämissäätiö and Emil Aaltonen Foundation. 


\section{References}

[1] R. Wyttenbach, A. Gallino, M. Alerci, F. Mahler, L. Cozzi, M. Di Valentino, J. J. Badimon, V. Fuster, R. Corti, Effects of percutaneous transluminal angioplasty and endovascular brachytherapy on vascular remodeling of human femoropopliteal artery by noninvasive magnetic resonance imaging, Circulation 110 (9) (2004) 1156-1161.

[2] J. R. Laird, Limitations of percutaneous transluminal angioplasty and stenting for the treatment of disease of the superficial femoral and popliteal arteries, Journal of Endovascular Therapy 13 (2_suppl) (2006) II-30-II-40.

[3] D. Xu, J. Li, L. Zou, Y. Xu, D. Hu, S. L. Pagoto, Y. Ma, Sensitivity and specificity of the ankle-brachial index to diagnose peripheral artery disease: a structured review, Vascular Medicine 15 (5) (2010) 361-369.

[4] V. Suominen, T. Rantanen, M. Venermo, J. Saarinen, J. Salenius, Prevalence and risk factors of pad among patients with elevated abi, European Journal of Vascular and Endovascular Surgery 35 (6) (2008) 709-714.

[5] S. Alatab, H. Fakhrzadeh, F. Sharifi, A. Mostashfi, M. Mirarefin, Z. Badamchizadeh, Y. Tagalizadehkhoob, Impact of hypertension on various markers of subclinical atherosclerosis in early type 2 diabetes, Journal of Diabetes \& Metabolic Disorders 13 (1) (2014) 1.

[6] M. T. Romanos, A. Raspovic, B. M. Perrin, The reliability of toe systolic pressure and the toe brachial index in patients with diabetes, Journal of foot and ankle research 3 (1) (2010) 31.

[7] J. Allen, A. Murray, Similarity in bilateral photoplethysmographic peripheral pulse wave characteristics at the ears, thumbs and toes, Physiological measurement 21 (3) (2000) 369.

[8] J. Allen, K. Overbeck, A. F. Nath, A. Murray, G. Stansby, A prospective comparison of bilateral photoplethysmography versus the ankle-brachial 
pressure index for detecting and quantifying lower limb peripheral arterial disease, Journal of vascular surgery 47 (4) (2008) 794-802.

[9] J. Allen, C. P. Oates, T. A. Lees, A. Murray, Photoplethysmography detection of lower limb peripheral arterial occlusive disease: a comparison of pulse timing, amplitude and shape characteristics, Physiological measurement 26 (5) (2005) 811.

[10] R. Erts, J. Spigulis, I. Kukulis, M. Ozols, Bilateral photoplethysmography studies of the leg arterial stenosis, Physiological measurement 26 (5) (2005) 865.

[11] M. Peltokangas, A. A. Telembeci, J. Verho, V. M. Mattila, P. Romsi, A. Vehkaoja, J. Lekkala, N. Oksala, Parameters extracted from arterial pulse waves as markers of atherosclerotic changes: performance and repeatability, IEEE Journal of Biomedical and Health InformaticsIn press. DOI: 10.1109/JBHI.2017.2679904.

[12] V. Jacomella, A. Shenoy, K. Mosimann, M. Kohler, B. Amann-Vesti, M. Husmann, The impact of endovascular lower-limb revascularisation on the aortic augmentation index in patients with peripheral arterial disease, European Journal of Vascular and Endovascular Surgery 45 (5) (2013) 497501.

[13] M. Peltokangas, A. Vehkaoja, J. Verho, M. Huotari, J. Rning, J. Lekkala, Monitoring arterial pulse waves with synchronous body sensor network, Biomedical and Health Informatics, IEEE Journal of 18 (6) (2014) 17811787. doi:10.1109/JBHI. 2014.2328788.

[14] M. Peltokangas, J. Verho, A. Vehkaoja, Night-time ekg and hrv monitoring with bed sheet integrated textile electrodes, Information Technology in Biomedicine, IEEE Transactions on 16 (5) (2012) 935-942.

[15] S. C. Millasseau, R. P. Kelly, J. M. Ritter, P. J. Chowienczyk, The vascular impact of aging and vasoactive drugs: comparison of two digital volume 
pulse measurements, American journal of hypertension 16 (6) (2003) 467472.

[16] M. Peltokangas, A. Vehkaoja, J. Verho, V. M. Mattila, P. Romsi, J. Lekkala, N. Oksala, Age dependence of arterial pulse wave parameters extracted from dynamic blood pressure and blood volume pulse waves, IEEE journal of biomedical and health informatics 21 (1) (2017) 142-149.

[17] V. Melenovsky, B. A. Borlaug, B. Fetics, K. Kessler, L. Shively, D. A. Kass, Estimation of central pressure augmentation using automated radial artery tonometry, Journal of hypertension 25 (7) (2007) 1403-1409.

[18] S. C. Millasseau, J. M. Ritter, K. Takazawa, P. J. Chowienczyk, Contour analysis of the photoplethysmographic pulse measured at the finger, Journal of hypertension 24 (8) (2006) 1449-1456.

[19] K. Takazawa, N. Tanaka, K. Takeda, F. Kurosu, C. Ibukiyama, Underestimation of vasodilator effects of nitroglycerin by upper limb blood pressure, Hypertension 26 (3) (1995) 520-523.

[20] J. Randolph, Free-marginal multirater kappa (multirater $\kappa$ free): An alternative to fleiss' fixed-marginal multirater kappa, in: Presented at the Joensuu Learning and Instruction Symposium, Vol. 2005, 2005.

[21] K. O. McGraw, S. P. Wong, Forming inferences about some intraclass correlation coefficients., Psychological methods 1 (1) (1996) 30.

[22] C. Lessells, P. T. Boag, Unrepeatable repeatabilities: a common mistake, The Auk (1987) 116-121.

[23] S. Ray, T. Buckenham, A. Belli, R. Taylor, J. Dormandy, The nature and importance of changes in toe-brachial pressure indices following percutaneous transluminal angioplasty for leg ischaemia, European journal of vascular and endovascular surgery 14 (2) (1997) 125-133. 
[24] L. Allouche-Cometto, P. Leger, H. Rousseau, D. Lefebvre, et al., Comparative of blood flow to the ankle-brachial index after iliac angioplasty, International angiology 18 (2) (1999) 154.

[25] H. Yokoyama, T. Shoji, E. Kimoto, K. Shinohara, S. Tanaka, H. Koyama, M. Emoto, Y. Nishizawa, Pulse wave velocity in lower-limb arteries among diabetic patients with peripheral arterial disease, Journal of atherosclerosis and thrombosis 10 (4) (2003) 253-258. 